\title{
Step Edge Selection during Ion Erosion of $\mathrm{Cu}(001)$
}

\author{
Peter Broekmann, Agnes Mewe, Herbert Wormeester,* and Bene Poelsema \\ MESA+ Research Institute, University of Twente, P.O. Box 217, 7500 AE Enschede, The Netherlands
}

(Received 19 March 2002; published 12 September 2002)

\begin{abstract}
Upon $\mathrm{Ar}^{+}$ion erosion of a $\mathrm{Cu}(001)$ surface, we observed with HR-LEED faceted structures on the surface oriented preferentially along $\langle 100\rangle$ at $200 \mathrm{~K}$ and along $\langle 110\rangle$ at $330 \mathrm{~K}$. These results are evaluated with a geometric rule, relating under kinetic conditions the orientation of the surviving steps to their advance rates. Application of this generic rule demonstrates that the $\langle 100\rangle$ steps advance slowest. This reveals enhanced interlayer mass transport across these steps. We also show that a transition from kinetically favored step orientation into the thermodynamically favored one occurs at very short time scales even near room temperature.
\end{abstract}

DOI: $10.1103 /$ PhysRevLett.89.146102

PACS numbers: 68.35.Fx, 68.03.Hj, 68.49.Jk, 68.55.Ac

Crystal growth and erosion of crystalline solids are governed by the formation of quite well defined planes under kinetic growth or erosion conditions. The final crystal shape is determined by those planes that have an extreme growth or erosion rate [1]. This generic textbook rule states that, for an expanding crystalline solid sphere, the planes with the lowest advancement rate determine the final shape, while for shrinking spheres those with the highest advancement rate survive. The expansion behavior described by this rule was shown to hold also in 2D for growing adatom islands on a $\mathrm{Pt}(111)$ surface. In this case the step edges with lowest advance rates survive. The difference in island shape observed at various temperatures was explained by the temperature dependence of the step advance rates [2]. This is the result of the difference in step edge diffusion of adatoms attached to steps. Note that the surface cleanliness has been shown to play a decisive role in the determination of the step advance rates, too [3]. The density of adatoms in front of a step edge can also vary due to interlayer mass transport over step edges with different diffusion barriers, depending on the decoration of the steps [3]. The kinetic growth situation described above should be distinguished from the situation in which thermodynamics govern the island shape. At sufficiently high temperatures, rearrangement processes can be very fast, and the islands assume equilibrium shapes with the domination of steps possessing the lowest line tensions. Under these conditions on a (001) oriented $\mathrm{Cu}$ surface, step edges with a $\langle 110\rangle$ orientation are expected and observed. In this Letter, we show the application of the generic geometric rule to the erosion of the $\mathrm{Cu}(001)$ surface. Figure 1(a) shows schematically that, if the lowest step edge advancement rate is that of the $\langle 110\rangle$ step edges, etch structures with closed packed step edges are expected. However, under kinetic conditions, i.e., at sufficiently low temperature, we observe eroded structures with step edges predominantly along the $\langle 100\rangle$ directions. The application of the geometric rule directly provides insight in the physics behind the emergence of differently oriented step orientations as indicated in
Fig. 1(b): the interlayer mass transport across $\langle 100\rangle$ oriented steps is much faster than that across the close packed $\langle 110\rangle$ steps, reducing the step advancement rate of $\langle 100\rangle$ step edges remarkably, leading to their survival during erosion.

Erosion through ion sputtering of a surface is complicated compared to growth by the fact that two different species relevant for the resulting morphology are created: (clusters of) vacancies and (clusters of) adatoms. Experimental results indicate that we can expect that, for an $800 \mathrm{eV} \mathrm{Ar}^{+}$ion bombardment of $\mathrm{Cu}(001)$, initially $2-3$ adatoms are created per atom that is physically removed from the surface [4,5]. Our STM measurements reveal that indeed adatom-clusters do evolve together with vacancy clusters. However, in all cases relevant to this Letter, a net etching occurs. This implies that vacancy islands always expand during ion bombardment. The influence of the adatoms on the morphology was discussed by Costantini et al. [5] for $\operatorname{Ag}(001)$. Their comparison between the structures observed after growth and erosion at low temperature led them to the conclusion that the adatoms created in the ion sputtering process play the dominant role in the morphology obtained after erosion. At elevated temperatures etch pits with step edges

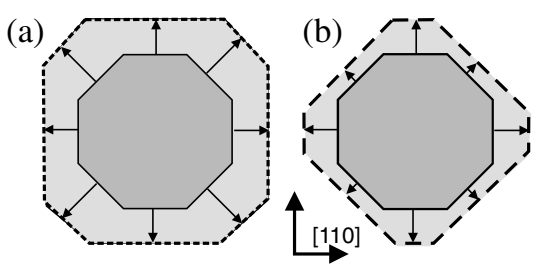

FIG. 1. Schematic picture of the change in island shape for different growth rates of step edges for (a) a vacancy island that expands through vacancy capture and (b) a vacancy island in which site selective interlayer mass current reduces the step advancement rate of the $\langle 100\rangle$ step edges. The length of the arrows show the net growth rate and the dotted lines indicate the changed morphology of the island, starting from an eightfold symmetric shape (solid lines). 
along $\langle 110\rangle$ azimuths were observed, comparable to previous $\mathrm{STM}$ measurements on $\mathrm{Cu}(001)[6,7]$. Previous to these measurements a "conventional" LEED study by Teichert et al. [8] on normal incidence ion bombardment on $\operatorname{Ag}(001)$ was performed. Their results suggested a $45^{\circ}$ difference in the azimuth orientation of defect structures for different preparation temperatures. Sputtering at elevated temperature $(300 \mathrm{~K})$ leads to structures with base lines parallel to the thermodynamically most stable $\langle 110\rangle$ directions, while ion bombardment at lower temperature $(170 \mathrm{~K})$ results in structures with step edges preferentially orientated along the less densely packed silver rows in $\langle 100\rangle$ directions [8].

Our experimental method of choice to characterize surface morphologies is high-resolution electron diffraction (HR- or SPA-LEED), with a resolution better than $0.1 \%$ of a Brillouin zone. All experiments have been conducted in ultrahigh vacuum (base pressure $<10^{-10}$ mbar). The investigated substrate is a $\mathrm{Cu}(001)$ surface which has been prepared anew before each experiment by $30 \mathrm{~min}$ of $800 \mathrm{eV} \mathrm{Ar}{ }^{+}$ion sputtering and subsequent annealing up to $800 \mathrm{~K}$ for $5 \mathrm{~min}$. A clean surface with an average terrace width exceeding $100 \mathrm{~nm}$ is yielded by this initial procedure. In the standard experiment the substrate temperature is quenched to $100 \mathrm{~K}$ immediately after switching off the ion beam. This quench takes about $1 \mathrm{~min}$ from 330 to $235 \mathrm{~K}$. Below $235 \mathrm{~K}$ no changes of the morphology occur on the time scale relevant in the experiment. Unless otherwise mentioned, $\mathrm{Ar}^{+}$ions with an energy of $800 \mathrm{eV}$ and an ion flux of $3 \times 10^{12}$ ions $\times \mathrm{s}^{-1} \mathrm{~cm}^{-2}$ were used to sputter the sample at normal incidence, corresponding to a sputter rate of about $0.16 \mathrm{ML} / \mathrm{min}$.

Figure 2 shows SPA-LEED profiles under slightly outof-phase conditions $\left(S_{z}=4.79\right)$ taken after ion erosion of $20 \mathrm{ML} \mathrm{Cu}$ at 200, 275, and $330 \mathrm{~K}$, respectively. It is immediately evident that ion bombardment at these temperatures followed by the above-mentioned standard quench procedure leads to a fundamentally different surface morphology.

After sputtering at elevated temperature $(330 \mathrm{~K})$, a fourfold symmetric spot profile is obtained with subspots aligned in the high symmetry $\langle 110\rangle$ axes. In contrast, the surface structuring at $200 \mathrm{~K}$ leads to a fourfold spot profile with subspots lying in the $\langle 100\rangle$ directions. For intermediate sputter temperatures $(275 \mathrm{~K})$, a more complex transition state is observed with intensity maxima in $\langle 310\rangle$ directions. By increasing the electron energy and thus going to an in-phase condition $\left(S_{z}=5\right)$, the subspots move to the center of the image consistently for all three temperatures. This implies that the observed diffraction patterns are related to facets rather than to a lateral ordering of defects as suggested in Ref. [8]. The presence of facet spots confirms the 3D character of these defects characterized by a relatively sharp terrace width distribution function. A quantitative analysis of the change in

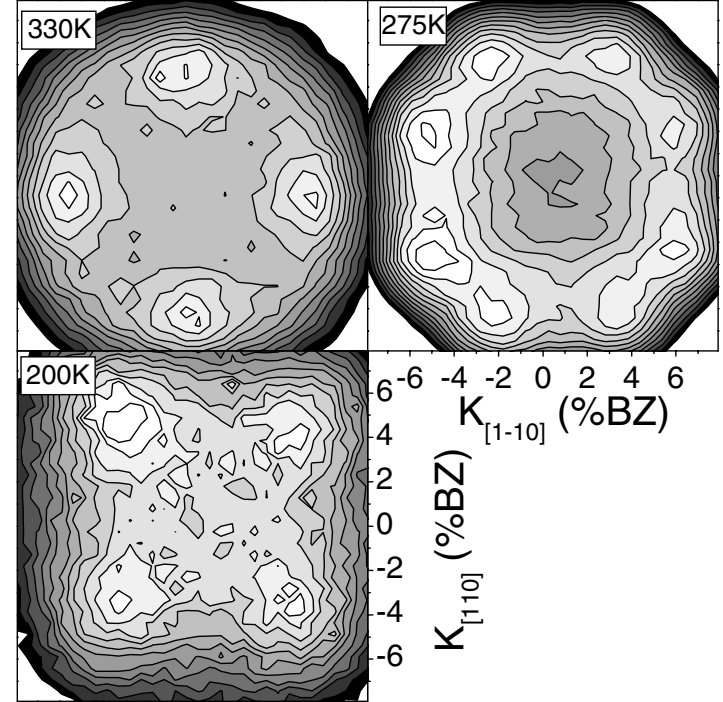

FIG. 2. SPA-LEED contour plots of the specular beam acquired after normal incidence $\mathrm{Ar}^{+}$sputtering of $20 \mathrm{ML} \mathrm{Cu}$ at 330,275 , and $200 \mathrm{~K}$. The peak profiles were obtained at $E=$ $267 \mathrm{eV}\left(S_{z}=4.79\right)$.

wave vector of these facet spots parallel to the surface, $k_{\|}$, as a function of the perpendicular wave vector, $k_{\perp}$, is presented in Fig. 3.

Facet angles of $19^{\circ}$ for $200 \mathrm{~K}$ and $17^{\circ}$ for $330 \mathrm{~K}$ are determined, corresponding to (103) and (115) facets, respectively. For $200 \mathrm{eV} \mathrm{Ar}^{+}$ions we found (117) facets at $330 \mathrm{~K}$, similar to Ernst [9] with $350 \mathrm{eV}$ ions. Within a temperature range from 330 to $400 \mathrm{~K}$ no change in the facet angle could be observed in our measurements. Also shown in Fig. 3 are idealized hardball models of the etch craters.

The erosion process for $\operatorname{Ag}(001)$ has also been studied by Teichert et al. [8] with kinetic Monte Carlo simulations. The erosion process was modeled by a single atom removal event, while the probability of diffusion paths

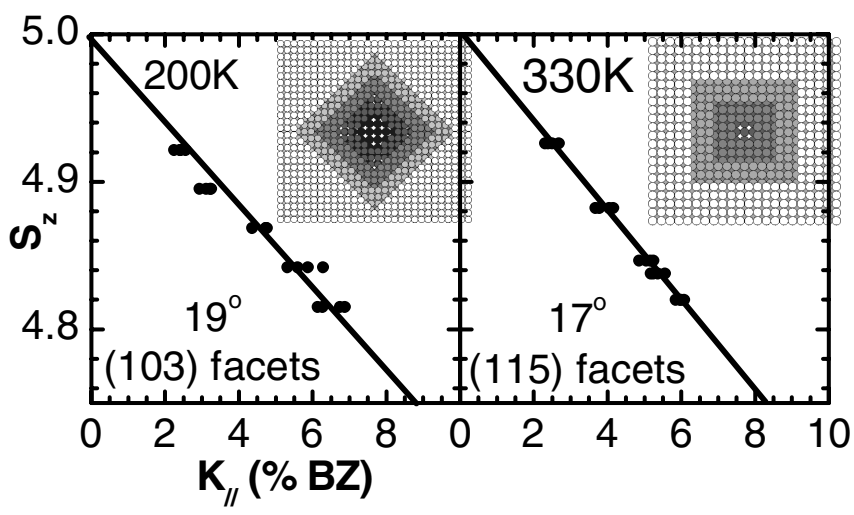

FIG. 3. Change in wave vector of the facet spots parallel to the surface, $k_{\|}$, as a function of the perpendicular scattering phase $S_{z}$; the insets represent idealized hardball models of the assumed defects. $200 \mathrm{~K}$ (left) and $330 \mathrm{~K}$ (right). 
was determined from first principle calculations. These simulations do reveal the morphological evolution at low and high temperatures we observed for the closely related $\mathrm{Cu}(001)$ surface. Site selective interlayer mass transport was identified as the responsible ingredient for the emerging surface morphology. The descent of adatoms is predominantly seen at certain "kink" sites, naturally present at $\langle 100\rangle$ rather than at close packed $\langle 110\rangle$ steps. The importance of highly selective interlayer diffusion paths for the surface morphology of $\mathrm{Cu}(001)$ was demonstrated recently by $\mathrm{Li}$ et al. [10] with STM. SPA-LEED data shows that already after $0.8 \mathrm{ML}$ erosion at $200 \mathrm{~K},\{103\}$ facets are present. Application of the geometric rule reveals that the step advancement rate of the $\langle 100\rangle$ oriented steps must be lower than that of the close packed $\langle 110\rangle$ ledges. This in spite of the fact that adatom diffusion along the latter steps is much faster than that along the more open ones. The latter feature is apparently counteracted by a phenomenon that efficiently reduces the growth rate in the $\langle 100\rangle$ directions. An interlayer mass transport current occurs preferentially across the $\langle 100\rangle$ step edges. The observed dominance of the $\langle 100\rangle$ step edges demonstrates that the advancement rate of $\langle 100\rangle$ step edges is smaller than the $\langle 110\rangle$ step edge advancement rate; see Fig. 1(b).

The different predominant step orientations observed after ion bombardment induced erosion at low and high temperature was attributed to fast post-erosion annealing effects at high temperatures by Teichert et al. Their simulations suggest that a less than $1 \mathrm{~s}$ anneal at room temperature suffices to change the surface morphology from a $\{10 n\}$ faceted structure to a $\{11 \mathrm{~m}\}$ faceted structure [8]: The transition from kinetically favored steps along $\langle 100\rangle$ to the thermodynamically favored steps along $\langle 110\rangle$ can be unexpectedly fast. In a recent experimental study, the possibility and thus the consequences of such fast annealing processes were disregarded [11]. However, exactly these events could well be responsible for the different orientations, observed at high and low temperature; see Fig. 2. In other words, the morphology observed after erosion at $330 \mathrm{~K}$ may be determined by a swift drive towards equilibrium while the $200 \mathrm{~K}$ data still represent the kinetic shape of the clusters. To check this issue experimentally, a quite different cooling procedure has been applied to avoid undesired annealing effects. We started quenching after an initial erosion of about $20 \mathrm{ML}$ of the $\mathrm{Cu}(001)$ surface at $330 \mathrm{~K}$ while the erosion was continued until a critical sample temperature of $235 \mathrm{~K}$ was reached. Subsequently, the quench was continued without sputtering down to $100 \mathrm{~K}$. During simultaneous sputtering and quenching from 330 to $235 \mathrm{~K}$, only $0.15 \mathrm{ML}$ of copper was additionally removed. Hence, the amount of additionally etched material is insignificant and we expect to obtain a morphology of the surface closely representing that during ion bombardment at $330 \mathrm{~K}$. The result of this experiment is given in
Fig. 4(a) and shows clearly a SPA-LEED profile indicative for facets with steps along the $\langle 100\rangle$ directions similar to the patterned surface at $200 \mathrm{~K}$ (Fig. 2). As a consequence, the orientation of step edges during sputtering is similar for temperatures between 200 to $330 \mathrm{~K}$. The deviations from this morphology while sputtering with the one recorded at 275 and $330 \mathrm{~K}$ (Fig. 2) are assigned straightforwardly to the annealing processes after the ion bombardment at elevated temperatures leading to a transition from a $\{10 n\}$ faceted structure to a $\{11 m\}$ faceted structure.

Obviously, the question whether during ion bombardment the kinetic cluster shape with $\langle 100\rangle$ oriented steps prevails or the thermodynamically favored $\langle 110\rangle$ steps is determined by the rate at which the system is driven towards equilibrium and the time scale set by the experimental conditions. Higher temperatures and lower erosion rates must lead to vacancy cluster geometries closer to the equilibrium shapes. An illustration of the latter is provided in Fig. 4(b). It exemplifies the diffraction profile of the $\mathrm{Cu}(001)$ surface after ion bombardment with a, compared to Fig. 4(a), 10 times reduced flux but equal fluence and also at $330 \mathrm{~K}$. The quenching procedure is the same as described for Fig. 4(a). In this case the amount of eroded material between the start of quench and stopping the sputtering is only $0.015 \mathrm{ML}$. The observed spot profile is similar to the one obtained after ion bombardment at $275 \mathrm{~K}$, i.e., indeed indicative of being closer to the equilibrium cluster shape, in line with the more efficient role of step edge diffusion during sputtering.

The application of the geometric rule provides straightforward evidence for enhanced interlayer diffusion across $\langle 100\rangle$ steps from the persistence of these steps even after etching as little as $0.8 \mathrm{ML}$ as discussed above. In contrast to this behavior, up to $0.5 \mathrm{ML}$, SPA-LEED and STM show that even at low temperature structures with $\langle 110\rangle$ step

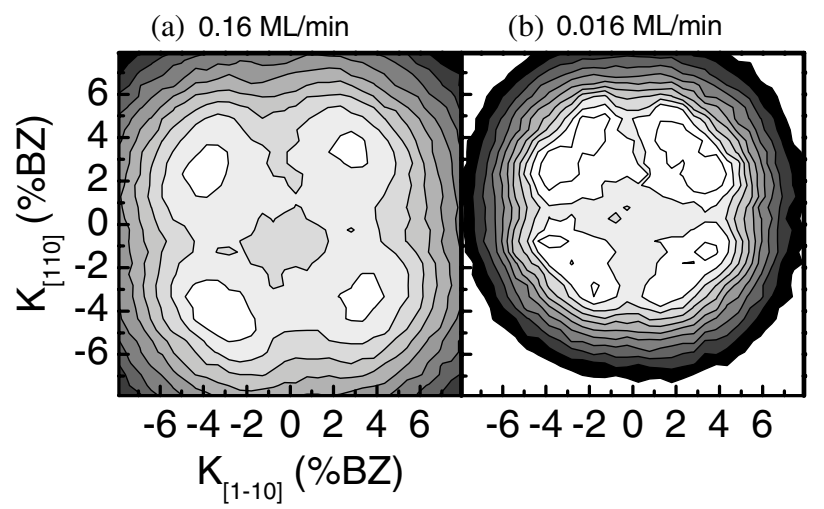

FIG. 4. SPA-LEED contour plot of the specular beam acquired after normal incidence $\mathrm{Ar}^{+}$sputtering of $20 \mathrm{ML} \mathrm{Cu}$ at $330 \mathrm{~K}$ with a sputter rate of (a) $0.16 \mathrm{ML} / \mathrm{min}$ and (b) $0.016 \mathrm{ML} / \mathrm{min}$. A different cooling procedure has been applied (for details, see text). The peak profiles were obtained at $E=271 \mathrm{eV}\left(S_{z}=4.81\right)$. 
edges dominate. STM reveals the reason for this remarkable observation in this coverage range more vacancy than adatom clusters are present and various vacancy and adatom clusters start to coalesce. The observation of dominant $\langle 110\rangle$ step edges indicates that under these conditions the selective interlayer diffusion across plays a subordinate role. The erosion is characterized by expanding vacancy clusters and advancement rates of the close packed steps will, similar to growth, be the dominant kinetic process: vacancy islands bounded by $\langle 110\rangle$ steps are observed; see Fig. 1(a). The observed lower interlayer mass transport during initial stages of erosion can be rationalized as follows: the adatoms generated as a result of ion impact diffuse, nucleate, and form initially expanding adatom islands. At the same time the created vacancies also diffuse, nucleate, and form expanding islands. Note that under the conditions relevant to our experiments, the integrated areas of the vacancy clusters always outweigh those of the adatoms clusters. Because of an always nonzero Ehrlich-Schwoebel barrier, the adatoms will initially be incorporated more easily in the adatom island rather than contribute to shrinking of the vacancy islands. With proceeding etching the vacancy islands become predominant and interlayer diffusion is increasing. The latter process takes place preferentially place across $\langle 100\rangle$ steps. We emphasize that during or just preceding coalescence paths with lower energy barriers become available too [8] further contributing to enhanced and site specific interlayer mass transport across $\langle 100\rangle$ ledges. So it appears that the reversal of the kinetic island orientation, occurring between etching of 0.5 and $0.8 \mathrm{ML}$, can be well accounted for in terms of site specific interlayer mass transport across the open $\langle 100\rangle$ step edges.

Extension of our findings for the ion bombardment induced erosion of $\mathrm{Cu}(001)$ to the closely related $\operatorname{Ag}(001)$ system provides a direct framework to understand the anomalous roughening behavior observed for the latter as reported by Costantini et al. [5]. They found that the width of the eroded vacuum- $\operatorname{Ag}(001)$ interface shows a pronounced maximum around $325 \mathrm{~K}$. By definition, the width of the surface morphology is intimately related to the probability for interlayer diffusion. An extrapolation of our observations for $\mathrm{Cu}(001)$ to the $\operatorname{Ag}(001)$ data leads to the following physical picture: At low temperatures $\langle 100\rangle$ oriented steps, allowing fast interlayer diffusion, prevail. Around and above room temperature the dominant ledge orientation becomes the thermodynamically favored $\langle 110\rangle$, causing reduced interlayer diffusion and rougher interfaces. With increasing temperature thermal excitations gradually suffice to overcome the Ehrlich- Schwoebel barrier, leading to in- creased interlayer mass transport and thus smoother interfaces. We do note that our model implies that the adatom islands growing on $\mathrm{Cu}(001)$ during normal incidence always have predominantly close packed steps. Only impurities, which can have an important impact on adatom island shapes [3], may alter this conclusion.

In conclusion, we have investigated the shape of the vacancy clusters on $\mathrm{Cu}(001)$ during ion bombardment induced erosion under kinetic limitations. The information is obtained from application of a well known rule, relating the presence of persisting step (facet) orientations to the lowest advancement (growth) rates. During the initial etching stages (up to $0.5 \mathrm{ML}$ ), the close packed $\langle 110\rangle$ ledges dominate. As soon as the surface is roughened to a stage at which interlayer diffusion becomes significant, the advancement rate of the $\langle 100\rangle$ steps is reduced due to the site selective descent of adatoms. It is even slowed down below that of the close packed $\langle 110\rangle$ ledges, making the $\langle 100\rangle$ ones dominate the surface morphology. We emphasize that, even at temperatures as low as room temperature, annealing can be unexpectedly fast, leading to dramatic changes of the surface morphology. We believe that our findings apply generically for nonreconstructed fcc(001) surfaces.

This work was possible due to a grant from the Alexander-von-Humboldt Foundation.

*Corresponding author

Electronic address: h.wormeester@tn.utwente.nl

[1] W. Kleber, Einführung in die Kristallographie (Verlag Technik GmbH, Berlin, 1990), 17th ed.

[2] T. Michely, M. Hohage, M. Bott, and G. Comsa, Phys. Rev. Lett. 70, 3943 (1993).

[3] M. Kalff, G. Comsa, and T. Michely, Phys. Rev. Lett. 81, 1255 (1998).

[4] T. Michely and C. Teichert, Phys. Rev. B 50, 11156 (1994).

[5] G. Costantini, F. Buatier de Mongeot, C. Boragno, and U. Valbusa, Phys. Rev. Lett. 86, 838 (2001).

[6] J. C. Girard, Y. Samson, S. Gauthier, S. Rousset, and J. Klein, Surf. Sci. 302, 73 (1994).

[7] M. Ritter, M. Stindtmann, M. Farle, and K. Baberschke, Surf. Sci. 348, 243 (1996).

[8] Ch. Teichert, Ch. Ammer, and M. Klaua, Phys. Status Solidi A 146, 223 (1994).

[9] H.-J. Ernst, Surf. Sci. 383, L755 (1997).

[10] M. Li, J. F. Wendelken, B.-G. Liu, E. G. Wang, and Z. Zhang, Phys. Rev. Lett. 86, 2345 (2001).

[11] G. Costantini, S. Rusponi, F. Buatier de Mongeot, C. Boragno, and U. Valbusa, J. Phys. Condens. Matter 13, 5875 (2001). 